\title{
O USO DO ENTEROCLISMA NO PREPARO PARA O PARTO: ANÁLISE DE SUAS VANTAGENS E DESVANTAGENS ${ }^{1}$
}

\author{
Maria Helena Baena de Moraes Lopes ${ }^{2}$ \\ Magda Aparecida Santos Silva ${ }^{3}$ \\ Fátima Filomena Mafra Christóforo ${ }^{4}$ \\ Deborah Cristina Junqueira de Andrade ${ }^{5}$ \\ Nara Regina Bellini ${ }^{5}$ \\ Rita de Cássia Cervi ${ }^{5}$ \\ Maria Clara Estanislau do Amaral ${ }^{6}$
}

Lopes MHBM, Silva MAS, Christóforo FFM, Andrade DCJ, Bellini NR, Cervi RC, et al. O uso do enteroclisma no preparo para o parto: análise de suas vantagens e desvantagens. Rev Latino-am Enfermagem 2001 novembro-dezembro; 9(6):49-55.

Investigaram-se os efeitos do enteroclisma sobre a contaminação fecal, durante o trabalho de parto e parto, entre mulheres atendidas num hospital-escola. A população foi constituída por 90 parturientes (43 partos normais, 27 fórceps e 20 cesáreas). Realizou-se um ensaio clínico randomizado, considerando-se dois grupos: com e sem enteroclisma. Pode-se concluir que o enteroclisma não abreviou o tempo de trabalho de parto e não reduziu a contaminação fecal. Entretanto, as mulheres que realizaram o enteroclisma tiveram opinião mais favorável sobre ele do que as que não o realizaram.

DESCRITORES: parto, parto normal, trabalho de parto, enema

\section{THE USE OF INTESTINAL CLEANSERS TO PREPARE FOR LABOR: ANALYSIS OF ADVANTAGES AND DISADVANTAGES}

This study aimed at investigating the effects of intestinal cleansers on fecal contamination during labor and delivery amongst women assisted in a university hospital. The subjects were 90 pregnant women (43 normal births, 27 forceps and 20 caesarean sections). The population was divided in two randomized groups: with and without the use of intestinal cleansers. It was possible to conclude that intestinal cleansers did not provide faster labor and did note reduce fecal contamination. However, the women who had used an intestinal cleanser tended to regard it as positive.

KEY WORDS: delivery, natural childbirth, labor, enema

\section{EL USO DE ENEMAS EN LA PREPARACIÓN PARA EL PARTO: ANALISIS DE SUS VENTAJAS Y DESVENTAJAS}

Se investigaron los efectos de los enemas sobre la contaminación fecal durante el trabajo de parto y el parto, entre mujeres atendidas en un hospital-escuela. La población estuvo constituida por 90 mujeres (43 partos normales, 27 con fórceps y 20 por cesárea). Se realizó un ensayo clínico aleatorio, se consideraron grupos: con y sin enemas. Se puede concluir que el enema no disminuyó el tiempo de trabajo de parto y no redujo la contaminación fecal. Aun así, las mujeres, que realizaron el enema tuvieron opinión más favorable sobre éste que aquellas que no lo realizaron.

DESCRIPTORES: parto, parto normal, trabajo de parto, enema

\footnotetext{
${ }^{1}$ Pesquisa financiada pelo Fundo de Apoio ao Ensino e à Pesquisa (FAEP) da Universidade Estadual de Campinas e pelo Programa PIBIC/CNPq; ${ }^{2}$ Enfermeiro, Professor Assistente Doutor, Faculdade de Ciências Médicas da Universidade Estadual de Campinas, endereço: Rua: Conceição, 552 - Ap. 25 - CEP 13010 050 - Campinas - São Paulo - Brasil; ${ }^{3}$ Ex-aluna e bolsista do Programa PIBIC/CNPq; ${ }^{4}$ Enfermeiro, Coordenadora do Programa de Educação Continuada do Centro de Atenção Integral à Saúde da Mulher (CAISM); ${ }^{5}$ Enfermeiro, Serviço de Enfermagem em Obstetrícia do CAISM; ${ }^{6}$ Enfermeiro. Faculdade de Ciências Médicas da Universidade Estadual de Campinas
} 


\section{INTRODUÇÃO}

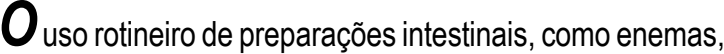
enteroclismas e supositórios, tem sido recomendado para facilitar a descida da apresentação fetal; estimular as contrações uterinas, abreviando desta forma o trabalho de parto; reduzir a contaminação durante o parto e, assim, diminuir o risco de infecção para a mãe e seu filho ${ }^{(1)}$

Entretanto, existem riscos potenciais no uso dos enemas, principalmente daqueles com sabão ("soapsuds"). Há casos documentados de irritação retal, colite, gangrena e choque anafilático ${ }^{(1)}$

Muitas mulheres têm um episódio de diarréia indolor e persistente até o primeiro período clínico do parto ${ }^{(2)}$. Observou-se que as mulheres que não fazem enema, mas relatam diarréia nas 24 horas que precedem a admissão ao hospital, apresentam menor freqüência de contaminação fecal durante o trabalho de parto do que as que não têm episódio de diarréia ${ }^{(2)}$.

$\mathrm{Na}$ literatura, há dois ensaios clínicos randomizados e controlados, cuja finalidade foi avaliar os efeitos do uso rotineiro de enemas na admissão de mulheres em trabalho de parto.

No primeiro ${ }^{(3)}$, os autores enfrentaram, durante 0 estudopiloto, a resistência das equipes médica e de enfermagem em desenvolvê-lo. Os médicos e enfermeiras acreditavam que a não realização do enema retardaria a descida da apresentação fetal, complicaria o trabalho de parto e levaria à contaminação fecal, principalmente no período expulsivo. Contudo, durante o desenvolvimento do estudo randomizado, as enfermeiras mudaram de opinião e passaram a fazer objeções quanto a realizar o enema, levando ao encerramento prematuro da pesquisa.

Assim, o estudo randomizado ${ }^{(3)}$ ficou constituído por 25 casos em cada grupo (experimental e controle). Os grupos não diferiram relativamente quanto aos índices de contaminação fecal, tanto no primeiro como no segundo períodos clínicos do parto. Esses autores analisaram os resultados combinados do estudo-piloto e do ensaio randomizado, num total de 149 mulheres que receberam o enema e de 125 mulheres que não o receberam, concluindo que não houve evidências de que o enema fosse prejudicial, mas sim de que causava estresse em algumas mulheres e, em muitas, desconforto. O uso de enema não diminuiu a incidência de contaminação fecal e de infecção, e não abreviou a duração do trabalho de parto. Por outro lado, as enfermeiras que assistiram ao parto notaram que, quando ocorreu contaminação fecal, as mulheres que tinham recebido 0 enema freqüentemente eliminavam fezes liquidas, o que dificultava a limpeza.

Um segundo estudo ${ }^{(4)}$, procurou reproduzir a mesma pesquisa, incluindo uma entrevista 24 horas após o parto, a fim de verificar como as mulheres sentiram-se em relação ao enema realizado durante o trabalho de parto. 0 grupo que recebeu o enema foi constituído por 109 mulheres; o que não o recebeu, por 113 mulheres. Esses autores concluíram que os resultados não evidenciaram a necessidade do uso rotineiro de enemas, posto que: 1) o uso de enema não abreviou a duração do trabalho de parto; 2) no segundo período clínico do parto, embora mais freqüentemente tenha ocorrido contaminação fecal no grupo que não recebeu 0 enema, as fezes geralmente eram formadas e em pequena quantidade, sendo facilmente removidas, e 3) 0 uso de enema não reduziu a incidência de infecção. Além desses achados, o estudo evidenciou que as mulheres reagiram favoravelmente à idéia de não ter que fazer o enema (grupo controle).

Os dois estudos mencionados ${ }^{(3-4)}$ trouxeram à tona aspectos importantes com relação ao uso rotineiro de enema nas mulheres em trabalho de parto. Os resultados obtidos indicaram a necessidade de personalizar $\mathrm{o}$ atendimento, permitindo que a cliente participe da decisão de se realizar ou não o procedimento. Contudo, nenhum deles foi conclusivo quanto à infecção materna, possivelmente em razão da baixa incidência de casos de infecção no puerpério.

No Centro de Atenção Integral à Saúde da Mulher (CAISM), em Campinas-SP, Brasil, onde se desenvolveu o presente estudo, a incidência de infecção puerperal encontra-se em torno de $2 \%$ ou menos (segundo a Comissão de Controle de Infecção Hospitalar), estando incluídos nesses índices tanto os casos de parto vaginal quanto os de cesárea. Por outro lado, os relatos da literatura apontam a cesárea como o fator predisponente mais importante para a infecção puerperal ${ }^{(5)}$. Assim, provavelmente os índices nos hospitais onde se desenvolveram as pesquisas citadas foram ainda menores, uma vez que não se incluíram as cesáreas.

De fato, no primeiro estudo mencionado ${ }^{(3)}$ houve apenas um caso de infecção em episiotomia, numa mulher que recebeu 0 enema; no segundo estudo ${ }^{(4)}$, não ocorreu nenhum caso de infecção em ferida cirúrgica perineal. Os dois trabalhos não fazem referência ao uso profilático de antibióticos, isto é, se houve diferença quanto à sua indicação entre os dois grupos, dado este que poderia alterar os resultados relativos à infecção materna.

No enteroclisma utiliza-se um volume maior de líquido ( 500 $\mathrm{ml}$ ou mais) do que no enema (cerca de $150 \mathrm{ml}$ ou menos). Devido a este fato, pode-se supor que o enteroclisma seja capaz de promover maior esvaziamento retal e, conseqüentemente, menor contaminação fecal durante o trabalho de parto, ainda que possa causar desconforto à parturiente. Um estudo realizado em nosso meio ${ }^{(6)}$ não encontrou diferença significativa entre os dois métodos (enteroclisma e enema), considerando-se apenas a contaminação fecal durante o parto.

Como até o momento existiam poucos trabalhos sobre 0 
uso do enteroclisma, e dado que no CAISM adotava-se, na rotina de esvaziamento intestinal, o enteroclisma, havia necessidade de validar os achados desses estudos em nosso meio, desenvolvendo trabalhos semelhantes que considerassem não somente a presença ou ausência de contaminação fecal, mas também a opinião das mulheres quanto à sua realização.

O uso de enteroclisma poderia levar a resultados diferentes quanto a contaminação fecal, principalmente no período expulsivo. Por outro lado, caso se demonstrasse que o uso rotineiro de enteroclisma não trazia benefícios, dever-se-ia indicá-lo somente após uma avaliação criteriosa da sua necessidade. Isto traria não só resultados positivos em termos de menor desconforto à mulher, como redução de custos.

\section{OBJETIVOS}

Geral: Investigar os efeitos do uso de enteroclisma sobre a contaminação fecal, durante o trabalho de parto e parto, entre mulheres atendidas no CAISM - Universidade Estadual de Campinas (UNICAMP).

Específicos:

- Verificar se o uso de enteroclisma abrevia a duração do trabalho de parto.

- Averiguar a associação entre o uso de enteroclisma e a contaminação fecal do períneo, no primeiro e segundo períodos clínicos do parto.

- Verificar se existe associação entre o uso de enteroclisma em parturientes e a infecção puerperal e neonatal.

- Verificar se o uso do enteroclisma altera a freqüência de indicação de antibioticoprofilaxia.

- Investigar a percepção e opinião das mulheres quanto ao uso de enteroclisma no preparo para o parto.

- Verificar se a sensação dolorosa das contrações uterinas, durante a realização do enteroclisma, está associada à opinião da mulher sobre o seu uso.

- Verificar a associação entre a contaminação fecal no primeiro e segundo períodos clínicos do parto e a eliminação espontânea de fezes antes da admissão hospitalar (eliminação prévia de fezes).

\section{MÉTODOS}

O estudo foi realizado nos setores de Pronto Atendimento, Centro Obstétrico e Alojamento Conjunto do CAISM - UNICAMP. Foram incluídas todas as mulheres em trabalho de parto, admitidas com indicação a priori de parto normal, que apresentassem gestação única de 37 semanas ou mais, bolsa íntegra e colo dilatado até no máximo $5 \mathrm{~cm}$.

Foram excluídas as mulheres com intercorrências clínicas e (ou) obstétricas, tais como diabetes, AIDS, doenças hipertensivas, síndromes hemorrágicas, gestação pós-termo e cardiopatias, as quais estão freqüentemente associadas a indicação de cesárea, indução/ condução ocitócica do parto ou imunossupressão.

Os dados das mulheres que atendiam aos critérios acima, mas que posteriormente evoluíram para cesárea, fórceps ou condução ocitócica, foram analisados apenas em relação a alguns aspectos, como a percepção e opinião sobre o uso de enteroclisma e outros, nos quais o tipo de parto não interferiria nos resultados.

Às mulheres, que atenderam aos critérios e concordaram por escrito em participar do estudo, explicou-se que poderiam ou não receber o enteroclisma, dependendo do grupo do qual fariam parte (experimental ou controle). A alocação aos grupos (com e sem enteroclisma) foi feita de acordo com o último dígito do número da ficha de atendimento do Serviço (Pronto Atendimento), considerandose: 'grupo com enteroclisma' (grupo experimental) quando ímpar e 'grupo sem enteroclisma' (grupo controle) quando par.

O grupo experimental recebeu o enteroclisma, que consistiu na aplicação de $500 \mathrm{ml}$ de solução glicerinada através de sonda retal. A mulher permaneceu em decúbito lateral esquerdo durante a administração do líquido, e assim permaneceu até sentir desejo imperioso de evacuar, sendo então encaminhada ao sanitário.

Durante o parto, a equipe de enfermagem do Centro Obstétrico preencheu uma ficha. As enfermeiras do Centro Obstétrico foram treinadas para este procedimento antes de se iniciar o estudo e os instrumentos foram pré-testados.

Entre 24 e 48 horas após o parto, completaram-se os dados desta ficha quanto à indicação de antibióticos e outras informações. A seguir, aplicou-se um questionário, com o objetivo de investigar a opinião e percepção da mulher em relação ao uso de enteroclisma durante o trabalho de parto.

Por ocasião da alta, agendou-se um retorno para a mulher após 10 a 15 dias de puerpério. Nessa ocasião foi verificado se houve desenvolvimento de algum processo infeccioso no coto umbilical ou ferida cirúrgica do períneo (episiotomia ou rotura) através de anamnese e exame físico. As mulheres que não compareceram ao retorno puerperal receberam visita domiciliar. Como em alguns casos não se conseguiu localizar a residência, quando transcorridos mais de 30 dias da data do parto, os dados foram obtidos por contato telefônico, investigando se a puérpera referia sinais/sintomas de infecção ou procura de Serviços de Saúde motivada por intercorrências.

Para estudar as diferenças entre os grupos utilizaram-se 
os testes estatísticos de Qui-quadrado ou, quando indicado, o Teste Exato de Fischer, com nível de significância de $5 \%{ }^{(7)}$. A análise dos dados foi feita separadamente para mulheres primíparas e multíparas.

A pesquisa foi aprovada pelas Comissões de Pesquisa e de Ética do CAISM, e as mulheres elegíveis que aceitaram participar assinaram um termo de consentimento livre e esclarecido.

\section{RESULTADOS}

A amostra foi constituída por 90 parturientes (43 partos normais, 27 fórceps e 20 cesáreas), que atendiam aos critérios de inclusão e concordaram em participar da pesquisa.

Considerando-se somente as mulheres que evoluíram para parto normal (duas primíparas e 20 multíparas no grupo com enteroclisma e quatro primíparas e 17 multíparas no grupo sem enteroclisma), verificou-se que o uso de enteroclisma, tanto em primíparas quanto em multíparas, não abreviou a duração do trabalho de parto, que foi de até $12 \mathrm{~h}$ para 16 parturientes $(72,7 \%)$ do grupo com enteroclisma e $15(71,4 \%)$ do grupo sem enteroclisma.

Analisando-se apenas o 'período expulsivo', também não houve diferença entre os dois grupos. A duração foi de até 20 minutos para $19(83,4 \%)$ parturientes do grupo com enteroclisma e 17 (81\%) do grupo sem enteroclisma.

Quanto à eliminação de fezes no primeiro e segundo períodos clínicos, considerando-se todos os partos por via vaginal (parto normal e fórceps, $n=70$ ), constatou-se que não houve diferença significativa entre os dois grupos, com e sem enteroclisma (período de dilatação: $\chi_{\text {corr }}^{2}=1,267, p=0,26$; período expulsivo: $\chi_{\text {corr }}^{2}=0,513$, $p=0,47)$.

Dentre as 43 mulheres que participaram do estudo e tiveram parto normal, 23 (14 pertencentes ao grupo com enteroclisma e nove do grupo sem enteroclisma) retornaram para o exame após o parto ou foram entrevistadas (e examinadas) durante as visitas domiciliares. Quando isto não foi possível, os dados foram obtidos por contato telefônico. As demais não foram avaliadas porque não puderam ser localizadas ou não tinham telefone.

No grupo 'com enteroclisma' os dados referentes ao período puerperal foram obtidos dentro do período de 12 a 56 dias após 0 parto; no grupo 'sem enteroclisma', de nove a 52 dias. Em sete casos (cinco do grupo com enteroclisma e dois sem) realizou-se visita domiciliária.

À avaliação, nenhuma mãe ou recém-nascido apresentou infecção relacionada ao parto via vaginal. Houve três casos em que a cliente referiu febre por mais de dois dias no puerpério (uma do grupo com enteroclisma e duas do grupo sem), sendo que duas delas (uma de cada grupo) foram submetidas à laqueadura tubária transabdominal após o parto e referiram sinais de inflamação apenas no local da incisão cirúrgica. A terceira mulher referiu ter tido febre por 14 dias, mas não mediu a temperatura.

Houve indicação mais freqüente de antibioticoprofilaxia nos casos de contaminação fecal no período expulsivo. Isto ocorreu apenas no grupo sem enteroclisma $(p=0,0119$, pelo Teste Exato de Fisher). Observou-se também que em 28 casos $(66,7 \%)$ houve uso de antibioticoprofilaxia (Figura 1).

INDICAÇÃO DE ANTIBIOTICOPROFILAXIA E CONTAMINAÇÃO FECAL

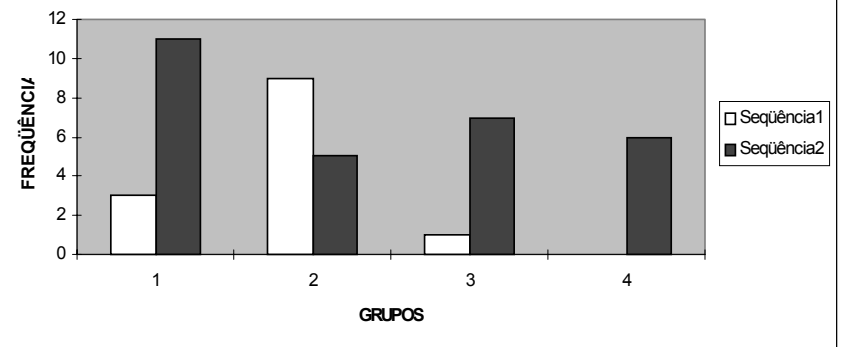

1 = COM indicação de antibioticoprofilaxia e COM uso de enteroclisma; 2 = COM indicação de antibioticoprofilaxia e SEM uso de enteroclisma; 3 = SEM indicação de antibioticoprofilaxia e COM uso de enteroclisma; 4 = SEM indicação de antibioticoprofilaxia e SEM uso de enteroclisma

Seqüência $1=$ com contaminação fecal

Seqüência 2 = sem contaminação fecal

Figura 1 - Distribuição das parturientes, com parto normal, segundo a indicação de antibioticoprofilaxia, considerando-se a contaminação fecal no período expulsivo e a realização de enteroclisma $(n=42$, porque em um caso não foi possível obter a informação sobre o uso de antibióticos)

Considerando-se o número total de mulheres que participaram do estudo ( $n=90$ ), o que inclui tanto os partos normais quanto os operatórios, 56 mulheres fizeram uso do enteroclisma e 34, não. Todas foram entrevistadas quanto à percepção e opinião sobre o seu uso. Dentre as primíparas que fizeram o enteroclisma, sete $(36,8 \%)$ consideraram o procedimento como experiência positiva; para oito $(42,1 \%)$ foi indiferente e para quatro $(21,1 \%)$ foi negativa. Já entre as multíparas, um maior percentual considerou positivo o uso de enteroclisma (18 ou 48,6\%) por sentirem-se mais aliviadas e seguras em não evacuar durante o parto; para oito $(21,6 \%)$ foi indiferente e para $11(29,7 \%)$ foi negativa, ainda que duas delas considerassem 0 procedimento 'necessário'. Uma das mulheres, entrevistada 22 dias após o parto, disse que 0 uso de enteroclisma evitou a flatulência após o parto, o que não ocorreu em partos anteriores, quando não fez a lavagem intestinal (era seu quarto filho). As mulheres que consideraram a experiência ruim, relataram eventos negativos como: dor, náusea, frio, desconforto, constrangimento e estranheza.

No grupo sem enteroclisma, oito $(57,1 \%)$ primíparas referiram sentir- 
se aliviadas por não o terem realizado; três $(21,4 \%)$ consideraram 0 fato indiferente e duas (14,3\%) tiveram percepção negativa pelo medo de evacuar durante o parto, apenas uma não respondeu. Quanto às multíparas, oito (40\%) mulheres referiram alívio pelo fato de não terem sido submetidas ao enteroclisma; para sete (35\%) delas foi indiferente - algumas alegavam que o mais preocupante no momento do parto era a dor das contrações; três (15\%) consideraram a experiência negativa; uma apresentou percepções positivas e negativas: achou bom não ter realizado a lavagem, mas teve medo de evacuar na sala de parto; uma não respondeu.

Quanto a fazer ou não o enteroclisma, caso a mulher pudesse optar, a opinião das mulheres variou de acordo com o grupo a que pertenciam, independente da paridade. No grupo com enteroclisma, 17 (89,5\%) primíparas e $33(89,2 \%)$ fariam o enteroclisma novamente. Frases como: Me senti bem aliviada quando fui para a mesa e não tive medo de fazer cocô na hora do parto, demonstram que o enteroclisma como preparo para o parto repercutiu de forma positiva, porque tinham medo ou constrangimento de evacuar em sala (Tabela 1).

Tabela 1 - Respostas das mulheres à pergunta: "Se pudesse escolher fazer ou não a lavagem intestinal, a senhora: faria, não faria ou não sabe. Por quê?", conforme a paridade - GRUPO COM ENTEROCLISMA

\begin{tabular}{|c|c|c|c|c|c|}
\hline \multirow[t]{2}{*}{ Hotivo } & \multicolumn{3}{|c|}{ Primipara } & \multicolumn{2}{|c|}{ Hultipara } \\
\hline & Faria & Nã̃o Faria & Nẫo Sabe & Faria & Nẫo Faria \\
\hline $\begin{array}{l}\text { Foi bom ter feito o } \\
\text { enteroclisma }\end{array}$ & 14 & 0 & 0 & 30 & 0 \\
\hline $\begin{array}{l}\text { Foi nuim ter feito o } \\
\text { enteroclisma }\end{array}$ & 1 & 0 & 1 & 0 & 3 \\
\hline $\begin{array}{l}\text { É nuim, mas neces- } \\
\text { sário }\end{array}$ & 1 & 0 & 0 & 0 & 0 \\
\hline $\begin{array}{l}\text { É uma rotina hospi- } \\
\text { talar }\end{array}$ & 1 & 0 & 1 & 3 & 0 \\
\hline Nỗo respondeu & 0 & 0 & 0 & 0 & 1 \\
\hline TOTÁL & 17 & 0 & 2 & 33 & 4 \\
\hline
\end{tabular}

No grupo que não fez o enteroclisma, a opinião das mulheres dividiu-se entre optar ou não pelo enteroclisma, sendo que cinco $(35,7 \%)$ primíparas e nove (45\%) multíparas optariam por fazer o enteroclisma. Para aquelas que desejavam realizar o procedimento, o motivo era o medo de evacuar no momento do parto. 0 motivo apresentado para não realizá-lo foi que a lavagem intestinal era incômoda, constrangedora (Tabela 2).
Tabela 2 - Respostas das mulheres à pergunta: "Se pudesse escolher fazer ou não a lavagem intestinal, a senhora: faria, não faria ou não sabe. Por quê?", conforme a paridade - GRUPO SEM ENTEROCLISMA

\section{$\begin{array}{lll}\text { Opinião } & \text { Primipara } & \text { Multipara }\end{array}$}

Fạ̛a Nầo Faria NầoSabe Fa̛⿱a Nẫo Faria NầoSabe

\begin{tabular}{|c|c|c|c|c|c|c|}
\hline $\begin{array}{l}\text { Foi bom nỗo ter feito } \\
\text { o erterocisma }\end{array}$ & 0 & 6 & 0 & 0 & 7 & 0 \\
\hline $\begin{array}{l}\text { Nẫo foi bom nẫo ter } \\
\text { feitoo enterodisma }\end{array}$ & 4 & 0 & 0 & 6 & 0 & 0 \\
\hline $\begin{array}{l}\text { Foi bom, masé ne- } \\
\text { cessário fazer o } \\
\text { enteroclisma }\end{array}$ & 0 & 0 & 1 & 1 & 1 & 0 \\
\hline $\begin{array}{l}\text { Éuma rotina hospta- } \\
\text { lar fazer o entero- } \\
\text { cisma }\end{array}$ & 1 & 0 & 0 & 2 & 0 & 0 \\
\hline Nẫorespondeu & 1 & 0 & 1 & 0 & 1 & 2 \\
\hline TOTAL & 6 & 6 & 2 & $\mathbf{9}$ & 9 & 2 \\
\hline
\end{tabular}

Analisando-se a opinião que a mulher teve sobre o uso de enteroclisma (considerando-se a opção por realizá-lo ou não novamente) e a sensação dolorosa das contrações uterinas no momento de admissão ao hospital, ocasião em que foi submetida (ou não) ao procedimento, não houve associação entre essas variáveis $\left(\chi_{(3)=}^{2} 3,443, p=0,3282\right)$.

Incluindo-se na análise todas as mulheres que realizaram parto vaginal (normal e fórceps), tanto primíparas quanto multíparas, verificou-se que a eliminação prévia de fezes (antes da admissão ao hospital), quando recente, levou a uma menor contaminação fecal no trabalho de parto e parto. A diferença é significativa $(p<0,05)$ apenas quando se considera o intervalo de zero a seis horas, no período de dilatação. Na Tabela 3 são apresentadas a freqüência de contaminação fecal, no primeiro período clínico do parto (período de dilatação) e a eliminação prévia de fezes.

Tabela 3 - Distribuição das parturientes segundo a contaminação fecal no período de dilatação e eliminação de fezes antes da admissão hospitalar

Eliminação Com Enteroclisma Sem Enteroclisma
prévia

(horas) Evacuou Não Evacuou Evacuou Não Evacuou

\begin{tabular}{lcccc}
\hline $0-6$ & 2 & 12 & 0 & 8 \\
$7-12$ & 8 & 7 & 2 & 6 \\
$13-24$ & 2 & 1 & 2 & 1 \\
Nầ Evacuou & 0 & 2 & 0 & 2 \\
Ignorado & 7 & 0 & 8 & 0 \\
ToTaL & $\mathbf{1 9}$ & $\mathbf{2 2}$ & $\mathbf{1 2}$ & $\mathbf{1 7}$ \\
\hline
\end{tabular}


A freqüência de contaminação fecal no segundo período clínico do parto (período expulsivo) e a eliminação prévia de fezes são apresentadas na Tabela 4.

Tabela 4 - Distribuição das mulheres segundo a eliminação de fezes antes da admissão hospitalar e a ocorrência de evacuação no período expulsivo - grupo experimental e controle

\begin{tabular}{lcccc}
\hline $\begin{array}{c}\text { Eliminąão } \\
\text { prévia } \\
\text { (horas) }\end{array}$ & \multicolumn{2}{c}{ Com Enteroclisma } & \multicolumn{2}{c}{ Sem Enteroclisma } \\
\hline $0-6$ & 3 & 12 & 1 & 8 \\
$7-12$ & 6 & 13 & 1 & 9 \\
$13-24$ & 2 & 2 & 3 & 3 \\
Nẫo Evacuou & 0 & 3 & 1 & 1 \\
lgnorado & 0 & 0 & 2 & 0 \\
TOTăL & $\mathbf{1 1}$ & $\mathbf{3 0}$ & $\mathbf{8}$ & $\mathbf{2 1}$ \\
\hline
\end{tabular}

\section{DISCUSSÃO}

Os achados estão de acordo com aqueles evidenciados pela literatura. Foi possível constatar que o uso do enteroclisma não abreviou o tempo de trabalho de parto e, portanto, a indicação de enteroclisma por crédito da diminuição do tempo de trabalho de parto necessita ser revista.

O estudo realizado no Brasil ${ }^{(6)}$, já citado no início deste artigo, comparou dois métodos de esvaziamento intestinal (enema e enteroclisma) e verificou que ambos se comportaram de forma semelhante. Contudo, o grupo que utilizou o enema apresentou, significativamente, menor número de evacuações após seu uso. Segundo esse estudo, o enema causa menos desconforto para mulher e, ainda, maior facilidade de introdução da solução por parte do profissional que executa o procedimento.

Observou-se, em nosso estudo, que a utilização de antibioticoprofilaxia foi freqüente nos dois grupos. Entretanto, a frequêencia de indicação foi maior no grupo sem enteroclisma com eliminação fecal no período expulsivo. Este fato é relevante, porque a eliminação fecal por si só não justifica a antibioticoprofilaxia; por outro lado, o uso de antibióticos pode interferir na ocorrência de infecção após o parto. Em vista disto, recomenda-se que em estudos semelhantes ao por nós desenvolvido investigue-se o uso profilático de antibióticos.

Houve tendência das mulheres (primíparas e multíparas) que realizaram o enteroclisma em optar por fazê-lo novamente. Entretanto, as que não foram submetidas ao procedimento (grupo controle) tiveram opinião dividida entre fazê-lo ou não. Algumas relataram medo de evacuar em sala; outras acharam bom não tê-lo realizado, ou referiram experiências negativas com o seu uso em partos anteriores.
Contrariamente ao esperado, não se observou qualquer relação entre sensação dolorosa das contrações uterinas no momento da realização do enteroclisma e a opção por fazê-lo em evento futuro (supunha-se que a realização do procedimento, na presença de contrações muito dolorosas, poderia levar a mulher a perceber a experiência como negativa).

Observou-se relação entre a eliminação prévia de fezes e menor contaminação fecal durante o parto apenas quando a evacuação foi recente (seis horas ou menos antes da internação). Recomenda-se que estudos com casuísticas maiores sejam realizados, a fim de confirmar estes achados.

De uma maneira geral, os motivos pelos quais o enteroclisma é indicado no preparo para o parto não foram justificados neste estudo, confirmando o que diz a literatura. As mulheres possuem muito medo de evacuar em sala de parto, submetendo-se ao procedimento mesmo que este lhe traga incômodo; este constrangimento deve ser tomado em consideração. Contudo, há outras mulheres que não desejariam realizar o enteroclisma novamente, pois percebem-no como ruim, desconfortável, constrangedor e julgam-no desnecessário, mesmo que venham a evacuar em sala.

Portanto, sugere-se uma reavaliação do uso do esvaziamento intestinal de rotina, analisando-se caso a caso, respeitando a opinião e a vontade da mulher, e indicando-o apenas quando houver ampola retal cheia, constatada pelo toque vaginal.

\section{CONCLUSÕES}

Do presente estudo pode-se concluir que: - O enteroclisma não diminuiu o tempo de trabalho de parto. - A freqüência da contaminação fecal no primeiro e segundo períodos clínicos do parto não diferiu significativamente entre os dois grupos (com e sem enteroclisma).

- Não foram observados casos de infecção puerperal nos dois grupos, mas o estudo não é conclusivo quanto a este aspecto.

- Houve maior freqüência de indicação de antibioticoprofilaxia no grupo sem enteroclisma que apresentou evacuação no expulsivo.

- A opinião das mulheres por realizar ou não o enteroclisma foi influenciada pelo grupo a que pertenciam; as que foram submetidas a ele tenderam a considerá-lo positivo.

- Não houve associação entre a opinião da mulher sobre o enteroclisma e sensação dolorosa das contrações uterinas.

- A evacuação prévia à admissão hospitalar, quando recente, diminuiu a freqüência de contaminação fecal durante o parto.

\section{Agradecimentos}

Agradecemos ao Fundo de Apoio ao Ensino e à Pesquisa (FAEP) Unicamp e ao Programa PIBIC/CNPq pelo auxílio financeiro, e às enfermeiras Rosane Ferreira Garcia, Alzira Cássia Simionato Costa e Marizete de Fátima Carvalho por colaborarem na coleta de dados. 


\section{REFERÉNCIAS BIBLIOGRÁFICAS}

1. Garforth S, Garcia J. Hospital admission practices. In: Chalmers I,

Enkin MJNC. Effective care in pregnancy and childbird. Oxford: Oxford

University Press; 1991. p.823-4.

2. Whitley N, Mack E. Are enemas justified for women in labor? Am J Nurs 1980; 1:1339.

3. Romney ML, Gordon H. Is your enema really necessary? Br Med J 1981; 282: 1269-71.
4. Drayton S, Rees $\mathrm{C}$. They know what they're doing. Nurs Mirror 1984; 159: 4-8.

5. Rezende J, Montenegro CAB. Infecção puerperal. In: Rezende J, Montenegro CAB. Obstetrícia Fundamental. 4. ed. Rio de Janeiro (RJ): Guanabara Koogan; 1984. p. 519-30.

6. Saito E. Estudo comparativo do uso de esvaziamento intestinal em parturientes. [dissertação]. São Paulo (SP): Escola de Enfermagem/USP; 1994.

7. Beiguelman B. Curso prático de bioestatística. 2 ed. Ribeirão Preto (SP): Revista Brasileira de Genética; 1991. 